\title{
Numerical modelling approach for the temperature dependent forming behaviour of Ti-6Al-4V
}

\author{
Thomas Papke ${ }^{1}$, Matthias Graser ${ }^{1}$, and Marion Merklein ${ }^{1}$ \\ ${ }^{1}$ Institute of Manufacturing Technology, Friedrich-Alexander-Universität Erlangen-Nürnberg, Egerlandstr. 13, 91058 Erlangen, Germany
}

\begin{abstract}
Titanium alloys offer several beneficial characteristics, such as high specific strength, metallurgical stability at elevated temperature, biocompatibility and corrosion resistance. With regard to these superior properties, Ti-6Al-4V is a commonly used titanium alloy for aerospace components and medical products. The production of parts made of Ti-6Al-4V can be done in various ways. One approach is forming at elevated temperature, which requires a focused design of parts, processes and numerical modelling of the forming process. Essential input parameters for the numerical models are temperature dependent material parameters. Since, the yield stress and Young's modulus of the material decrease significantly at elevated temperature, the forming limits are enhanced. For the characterization of the forming behaviour, uniaxial tensile tests at temperatures from $250{ }^{\circ} \mathrm{C}$ to $400{ }^{\circ} \mathrm{C}$ have been conducted. The samples are heated by conduction in a thermal-mechanical simulator for the tensile test. However, the resulting inhomogeneous temperature distribution along the longitudinal axis of the specimen is a challenge in order to measure proper material properties. Inhomogeneous temperature distribution leads to varying mechanical properties and temperature dependent forming behaviour. To overcome this issue, simple numerical models based on experimental data are necessary, which allow the estimation of the influence of the inhomogeneous temperature distribution. In this paper, therefore, the temperature distribution and the subsequent tensile test are investigated using electrical-thermal and mechanical numerical simulations of the tensile test at elevated temperature. With the combined approach of experimental tests and numerical simulations, the forming behaviour of Ti-6Al-4V can be modelled.
\end{abstract}

Keywords: Modelling; Thermal effects; Titanium

\section{Introduction}

The titanium alloy Ti-6Al-4V has become a popular material for medicine or aerospace applications. Beneficial material properties are biocompatibility, corrosion resistance and high specific strength. Despite of these advantages, narrow forming limits at room temperature of titanium are challenges for metal forming processes. Ti-6Al-4V consists of densely packed hexagonal $\alpha$ - and body-centred cubic $\beta$-phase. Due to the crystal structure of the $\alpha$-phase, the ductility is reduced, which leads to narrow forming limits at room temperature [1]. However, by forming at elevated temperatures, the yield stress decreases and the forming limits are enhanced [2]. In [3] experiments on Ti-6Al-4V sheet metal parts are conducted at temperatures below $600{ }^{\circ} \mathrm{C}$, which results in a significantly lower yield stress compared to forming at $20^{\circ} \mathrm{C}$. This indicates that forming of Ti-6Al-4V is also possible at elevated temperatures below the $\beta$-transus temperature [1].

A commonly used device for conducting hot tensile tests is the thermal-mechanical simulator Gleeble 3500 from Dynamic Systems Inc. (DSI). For testing at elevated temperatures, the specimen is clamped between copper grips and heated by conduction, which means the specimen is part of an electrical circuit and represents the resistance. However, the temperature distribution from the centre of the specimen to the clamping is not homogenous. The temperature distribution can be described by analytical equations [4]. One challenge resulting from the temperature gradient along the axis of the sample is the displacement of the forming zone relative to the zone of the target temperature. This influences the forming behaviour and thus leads to differing results regarding mechanical properties [5]. Since the test conditions according to DIN EN ISO 6892-2 [6] require a temperature deviation of less than $\pm 3{ }^{\circ} \mathrm{C}$ for test temperature lower than $600{ }^{\circ} \mathrm{C}$, the inhomogeneous temperature distribution is critical in order to gain valid testing results. An approach to ensure the failure of the specimen under tensile load at the area of target temperature is to reduce the gradient of temperature along the axis of the sample. Therefore, Ganapathy et al. [8] modified the shape of the specimen by adding extra copper wires at the edges of the specimen which leads to a significantly lower temperature gradient. However, the influences of the modified specimen geometry remain unclear.

It can be seen, that hot tensile tests in accordance with [6] are challenging. Hence, combined experimental

Corresponding author: thomas.papke@,fau.de 
and numerical methods are applied to determine material properties influenced by temperature gradients. Kardoulaki et al. [9] used the commercial software Abaqus to implement separate models for the electrothermal and mechanical simulation of a hot tensile test in a Gleeble testing system for free cutting steel (FCS). Furthermore, the implementation of a complex electrothermal-mechanical coupled simulation of the hot tensile test for 22MnB5 using Abaqus is shown by Gerhardt [10]. As a result, the forming behaviour from the experimental tensile test can be simulated by the coupled model. The electro-thermal modelling approach based on the experimental setup should be investigated for LSDyna in this work.

The aim of this paper is to analyse the influence of a temperature gradient on the forming behaviour of Ti-6Al-4V sheet metal parts by using a combined experimental and numerical approach. Since the tensile test is a simple forming test, the results could be used for the investigation of the influence of temperature gradients on sheet metal parts in more complex forming processes as deep drawing [11] or bending [12].

Within this work, the tensile test setup in a Gleeble 3500 for Ti-6Al-4V is modelled by using the electromagnetism module of LS-Dyna. The numerical model is divided into two subsequent models, an electrothermal for simulating the temperature distribution and a mechanical model for the tensile test. The models are not coupled, because the modelling approach should be considered as a separated analysis of both process steps. In the first step, an electro-thermal model is set up to simulate the temperature distribution along the sample's longitudinal axis. Based on the resulting temperatures, flow curves from experimental tensile tests are implemented into the mechanical model, which takes the temperature dependent flow behaviour into account. Prior to the simulations, experimental data of electrical current and flow curves are derived from tensile tests at elevated temperature with Ti-6Al-4V sheet metal parts.

\section{Experimental Setup}

\subsection{Tensile test}

For the tensile test at elevated temperature, a Gleeble 3500 from Dynamic Systems Inc. (DSI) is used. The machine is equipped with a $100 \mathrm{kN}$ load cell for the measurement of the forming force. Furthermore, for the optical strain measurement, an ARAMIS System from GOM is applied. For the measurement of the strain on the specimens' surface, the samples are covered with a stochastic pattern [3]. The change of the stochastic pattern is recorded by a CCD-Camera with $40 \mathrm{~Hz}$ and distortion of the pattern is used to calculate the true stress-true strain curve. The specimen geometry has a width of $12.5 \mathrm{~mm}$, a parallel length of $62.5 \mathrm{~mm}$ and a sheet thickness of $1.0 \mathrm{~mm}$. Since the test is focused on the forming behaviour at elevated temperature; the rolling direction (RD) for all specimens is $0^{\circ}$. The strain rate is $0.1 \mathrm{~s}^{-1}$. The specimens are clamped between a pair of copper grips without cooling, which is sufficient for the maximum temperature of $400{ }^{\circ} \mathrm{C}$ investigated within this work.

The temperature during the test is measured by up to four thermocouples type- $\mathrm{K} \mathrm{Ni} / \mathrm{Cr}-\mathrm{Ni}$, to investigate the temperature distribution along the longitudinal axis of the specimen. The first thermocouple is located in the centre of the specimen, whereas the others are placed along the longitudinal axis with $10 \mathrm{~mm}, 20 \mathrm{~mm}$ and $32.5 \mathrm{~mm}$ distance from the centre.

Before the tensile test, the samples are heated from room temperature to the testing temperature within $30 \mathrm{~s}$. This temperature is held for $15 \mathrm{~s}$, in order to ensure a consistent test condition. The tensile test is conducted subsequently as shown in Fig. 1.

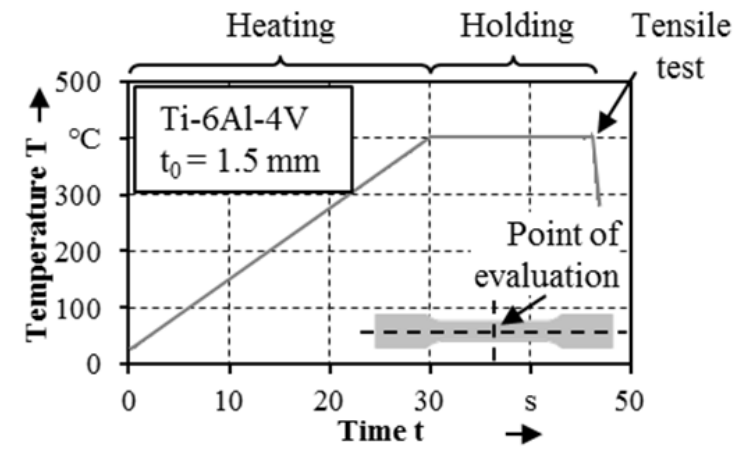

Fig. 1. Temperature-time curve for the tensile test

In addition to the tests at elevated temperature, tensile tests at room temperature $\left(20^{\circ} \mathrm{C}\right)$ are conducted in order to compare the forming behaviour. The tests are performed with a universal testing machine Zwick Z 100 Allround Line, which is equipped with a $100 \mathrm{kN}$ load cell. Like in the tensile test at elevated temperature, the strain on the surface is measured by an ARAMIS System. To avoid influences of the sample geometry, the same geometry as for the tensile test at elevated temperature is used.

\subsection{Electrical properties}

Besides the temperature distribution electrical current during the heating process is measured. The experimental setup is shown in Fig. 2.

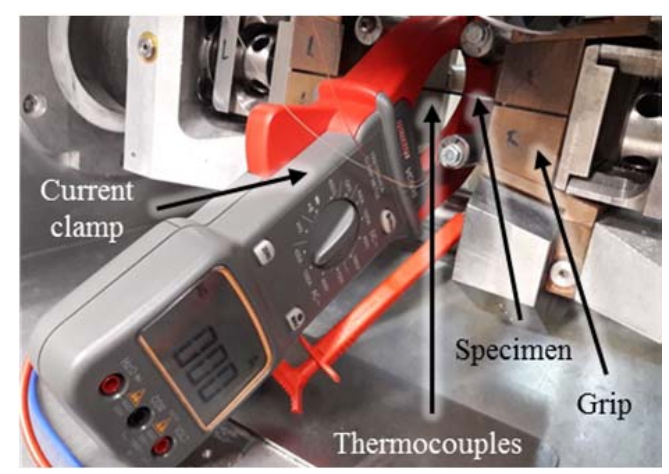

Fig. 2. Experimental setup for tensile test in Gleeble 3500

For measuring the current through the specimen, a current clamp VC605 from Voltcraft with a 
measurement range of $1000 \mathrm{~A}$ for $\mathrm{AC}$ is used, since the sample is heated by alternating current [10]. The result is the root mean square (RMS) value of the $\mathrm{AC}$, which is equal to the value of a direct current that would produce the same average power in the resistor.

\section{Numerical Model}

The modelling approach is based on two separate numerical models. The first model is used to simulate the temperature distribution in the specimen during the resistive heating process, whereas the second model is a mechanical model for the simulation of the tensile test. Based on the temperature distribution, which results from the electro-thermal model, the specimen is subdivided into several sections with different flow curves to simulate the tensile test with the mechanical model. For the numerical models, the specimen geometry from the experiments is used with a sheet thickness of $1.5 \mathrm{~mm}$.

\subsection{Electro-thermal Model}

For the electro-thermal model, the electromagnetism module [14] including resistive heating [15] from LS-Dyna is applied. The simulation model consists of two pairs of grips and the tensile specimen as shown in Fig. 3. The mesh of the specimen consists of constant stress solid hexahedral elements with a length of $0.5 \mathrm{~mm}$ in $\mathrm{z}$-direction and up to $1 \mathrm{~mm}$ in $\mathrm{x}$ - and $\mathrm{y}$-direction.

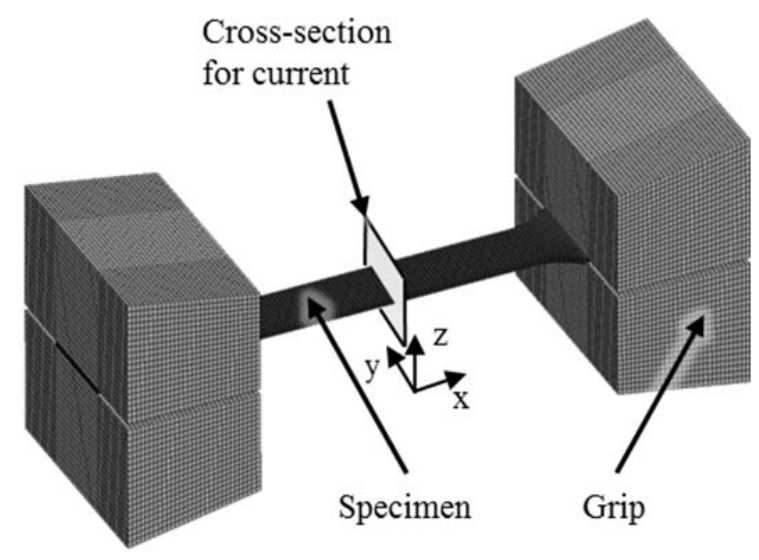

Fig. 3. Setup of the electro-thermal numerical model

The grips and the specimen are connected with nodes in the contact area. The maximum element size at the grips is $1.5 \mathrm{~mm}$, which is bigger than for the specimen to reduce the total number of elements and the temperature increase of the grips is neglected in this work. In total, the model consists of 157920 elements. As boundary conditions, the initial temperature for the grips and the specimen is set to $32{ }^{\circ} \mathrm{C}$, which is selected due to the temperature in the Gleeble process chamber. The material keyword MAT_T01 is used for all parts in the electro-thermal model. The material card allows thermal isotropic properties and includes constant values for specific heat (hc) and thermal conductivity (tc) [16]. The electrical current is imposed through the cross-section at the symmetry plane of the specimen and implemented by a current-time-curve. In table 1 mechanical, thermal and electrical properties are listed. The material properties for copper and Ti-6Al-4V are taken from literature. Parameters at room temperature are chosen in order to keep the numerical models simple.

Table 1. Properties of Copper $(\mathrm{Cu})$ and Ti-6Al-4V (Ti)

\begin{tabular}{|c|c|c|}
\hline Element & Style & Ref. \\
\hline \multirow{2}{*}{ Young's modulus } & $\mathrm{Cu}: 117 \mathrm{GPa}$ & {$[17]$} \\
& $\mathrm{Ti}: 110 \mathrm{GPa}$ & {$[1]$} \\
\hline \multirow{2}{*}{ Mass density } & $\mathrm{Cu}: 8950 \mathrm{~kg} / \mathrm{m}^{3}$ & {$[17]$} \\
& $\mathrm{Ti}: 4430 \mathrm{~kg} / \mathrm{m}^{3}$ & {$[1]$} \\
\hline \multirow{2}{*}{ Poisson ratio } & $\mathrm{Cu}: 0.35$ & {$[17]$} \\
& $\mathrm{Ti}: 0.342$ & {$[1]$} \\
\hline \multirow{2}{*}{ Specific heat capacity } & $\mathrm{Cu}: 400 \mathrm{~J} /(\mathrm{kgK})$ & {$[17]$} \\
& $\mathrm{Ti}: 546 \mathrm{~J} /(\mathrm{kgK})$ & {$[18]$} \\
\hline \multirow{2}{*}{ Thermal conductivity } & $\mathrm{Cu}: 400 \mathrm{~W} /(\mathrm{mK})$ & {$[17]$} \\
& $\mathrm{Ti}: 6.6 \mathrm{~W} /(\mathrm{mK})$ & {$[18]$} \\
\hline \multirow{2}{*}{ Electrical conductivity } & $\mathrm{Cu}: 58 \mathrm{e} 61 /(\Omega \mathrm{m})$ & {$[17]$} \\
& $\mathrm{Ti}: 5.8 \mathrm{e} 51 /(\Omega \mathrm{m})$ & {$[18]$} \\
\hline
\end{tabular}

\subsection{Mechanical Model}

The second part of the modelling approach uses a mechanical model for simulating the tensile test at elevated temperature. The aim is to investigate the forming behaviour in dependence of the temperature gradient of the experiment compared to ideal isothermal conditions at $400^{\circ} \mathrm{C}$. Based on the mesh of the electrothermal simulation, the maximum temperatures at the elements are evaluated and the specimen is subdivided into four major sections with different flow curves depending on the temperature. The assignment of flow curves from the experimental tensile tests is based on a range of temperatures, which is shown in table 2.

Table 2. Temperature range for flow curves

\begin{tabular}{|c|c|}
\hline Flow curve for section & Temperature range for section \\
\hline $\mathrm{FC}_{20}{ }^{\circ} \mathrm{C}$ & $20{ }^{\circ} \mathrm{C}-225{ }^{\circ} \mathrm{C}$ \\
\hline $\mathrm{FC}_{250}{ }^{\circ} \mathrm{C}$ & $226^{\circ} \mathrm{C}-300{ }^{\circ} \mathrm{C}$ \\
\hline $\mathrm{FC}_{360}{ }^{\circ} \mathrm{C}$ & $300{ }^{\circ} \mathrm{C}-380{ }^{\circ} \mathrm{C}$ \\
\hline $\mathrm{FC}_{400}{ }^{\circ} \mathrm{C}$ & $381{ }^{\circ} \mathrm{C}-500{ }^{\circ} \mathrm{C}$ \\
\hline
\end{tabular}

Since for the forming behaviour the target temperature $400{ }^{\circ} \mathrm{C}$ is investigated, a small temperature range for the subdivision is selected at high temperatures and wider at lower temperatures. Furthermore, an equidistant interval between the temperatures would include a linear temperature distribution along the x-axis, which is not the case in the experiment as shown in Fig. 6. The resulting subdivision of the mesh and the setup for the tensile test is shown in Fig. 4. In accordance with the tensile test experiments, one side of the specimen is clamped to avoid movement, whereas the other side is moved with $5 \mathrm{~mm} / \mathrm{s}$, where the tensile force is applied. The boundary conditions are implemented with an SPC 
keyword node set for the nodes at the outer side in the yz-plane.

Since the stress state is uniaxial tension; the material model is based on von Mises. The corresponding keyword in LS-Dyna is MAT_024, which is indicated as piecewise linear plasticity model with elasto-plastic material behaviour in LS-Dyna [16]. The material model includes the flow curves for $20^{\circ} \mathrm{C}, 250{ }^{\circ} \mathrm{C}, 360^{\circ} \mathrm{C}$ and $400{ }^{\circ} \mathrm{C}$, which result from the tensile tests in section 4.1. Besides flow curves, mass density, Young's modulus and Poisson's ration of table 2 are considered.

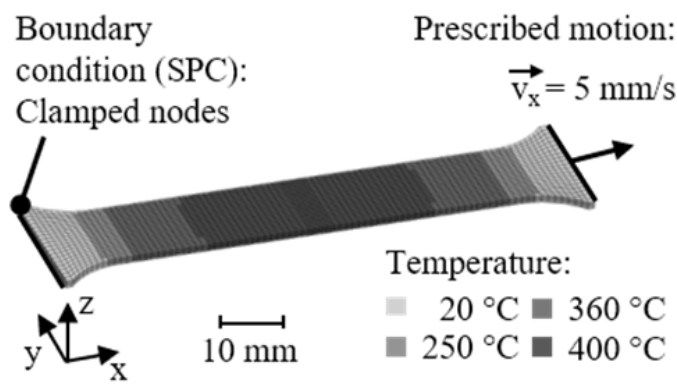

Fig. 4. Setup for the numerical model of the tensile test

\section{Results}

\subsection{Tensile test}

The tensile tests are conducted at $20{ }^{\circ} \mathrm{C}, 250{ }^{\circ} \mathrm{C}, 360{ }^{\circ} \mathrm{C}$ and $400{ }^{\circ} \mathrm{C}$, where the maximum true strain reach from 0.10 to 0.15 for all temperatures. However, for the numerical model in LS-Dyna flow curves with a true strain of 1.0 are necessary. Hence, the experimental data is extrapolated to higher true strains. Within this work, the model of Hockett-Sherby [21] is used. In prior investigations by Merklein and Schaub [3], the extrapolation models presented by Swift [19] and Nemat-Nasser [20] are applied. In comparison to Swift and Nemat-Nasser, the model by Hockett-Sherby comprises a lower hardening effect for higher true strain values. Essentially, the model is based on four parameters [21].

$$
\sigma(\varphi)=\sigma_{\mathrm{s}}-\left(\sigma_{\mathrm{s}}-\sigma_{\mathrm{y}}\right) \exp \left(-m \varphi^{p}\right)
$$

To calculate the flow curve, equation (1) includes $\sigma_{\mathrm{s}}$, representing the steady-state flow stress, the yield stress $\sigma_{y}$, which results from the tensile test and the free parameters $\mathrm{m}$ and $\mathrm{p}$. The parameters for calculating the flow curves are shown in table 3 .

Table 3. Used parameters for Hockett-Sherby extrapolation

\begin{tabular}{|c|c|c|c|c|}
\hline $\begin{array}{c}\text { Flow } \\
\text { curve }\end{array}$ & $\boldsymbol{\sigma}_{\mathbf{s}}$ in $\mathrm{MPa}$ & $\boldsymbol{\sigma}_{\mathbf{y}}$ in $\mathrm{MPa}$ & $\mathbf{m}$ & $\mathbf{p}$ \\
\hline $\mathrm{FC}_{20}{ }^{\circ} \mathrm{C}$ & 1300 & 900 & 1 & 0.2 \\
\hline $\mathrm{FC}_{250{ }^{\circ} \mathrm{C}}$ & 1000 & 713 & 1 & 0.2 \\
\hline $\mathrm{FC}_{360{ }^{\circ} \mathrm{C}}$ & 1200 & 600 & 3 & 0.7 \\
\hline $\mathrm{FC}_{400}{ }^{\circ} \mathrm{C}$ & 1000 & 600 & 3 & 0.7 \\
\hline
\end{tabular}

\subsection{Electrical measurement}

Besides the temperature, the electrical current is measured during the tensile test at elevated temperature to link thermal and electrical values. In Fig. 5 are shown the measured values for current and temperature in the centre of the specimen. The current increases during the heating process and drop significantly after $30 \mathrm{~s}$, when the holding phase starts. The increase in current during the heating phase could be explained by energy losses due to convection and radiation as well as temperature dependent physical properties. Hence, for an increase in temperature during the heating phase more current is needed.

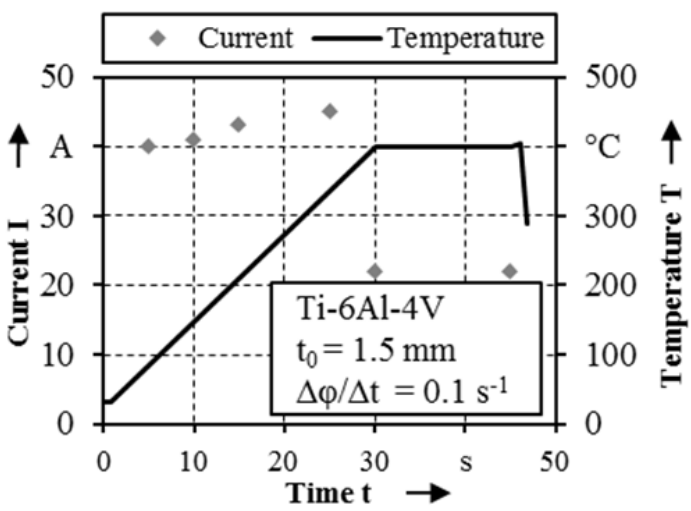

Fig. 5. Current and temperature for the tensile test at $400{ }^{\circ} \mathrm{C}$

\subsection{Numerical model}

The experimental data from the electrical measurement and the tensile tests are implemented in the numerical models. In the first step, the electro-thermal model is used for numerical modelling of the temperature distribution and the result compared to experimental data. Secondly, the mechanical model is set up to investigate the influence of inhomogeneous temperatures in a forming process demonstrated on a tensile test at elevated temperature. For the evaluation, the temperature distribution on the specimen after the heating process, the force-displacement curve and the strain distribution are analyzed. Finally, the mechanical numerical model is validated by experimental results.

\subsubsection{Electro-thermal model}

To evaluate the electro-thermal model, the temperature distribution along the $\mathrm{x}$-axis of the specimen is analysed. In accordance with the location of the thermocouples in the experiment, the elements at the surface of the specimen are investigated by a section plane. Since the specimen is symmetric, only one half of the sample is presented. In Fig. 6 the temperature distributions for experiment and simulation are shown for $t=30 \mathrm{~s}$, which represents the end of the heating process. In the simulation, the maximum temperature is reached after the heating process and a continuous temperature distribution is received, which is indispensable for implementing temperature dependent flow curves into the mechanical model. The experimentally measured 
temperature at the centre $(\mathrm{x}=0 \mathrm{~mm})$ is $400.5^{\circ} \mathrm{C}$, which is almost reached in the simulation with $399.2^{\circ} \mathrm{C}$. The deviation between the experimental values and calculated temperatures increases with higher distance from the centre. This indicates that the energy, which is introduced by the current is too small. Hence, the temperature in the simulation decreases faster for lower temperatures than in the experiment. Higher energy supply in the simulation could reduce the difference from the experimental data. The maximum deviation is about $17 \%$ at $x=32.5 \mathrm{~mm}$. One explanation could be that the material parameters implemented in the simulation are kept constant. The result could be improved by temperature dependent parameters. Therefore to improve the model it is mandatory to analyse the physical properties of the sheet material used in this work and compare them to literature values.

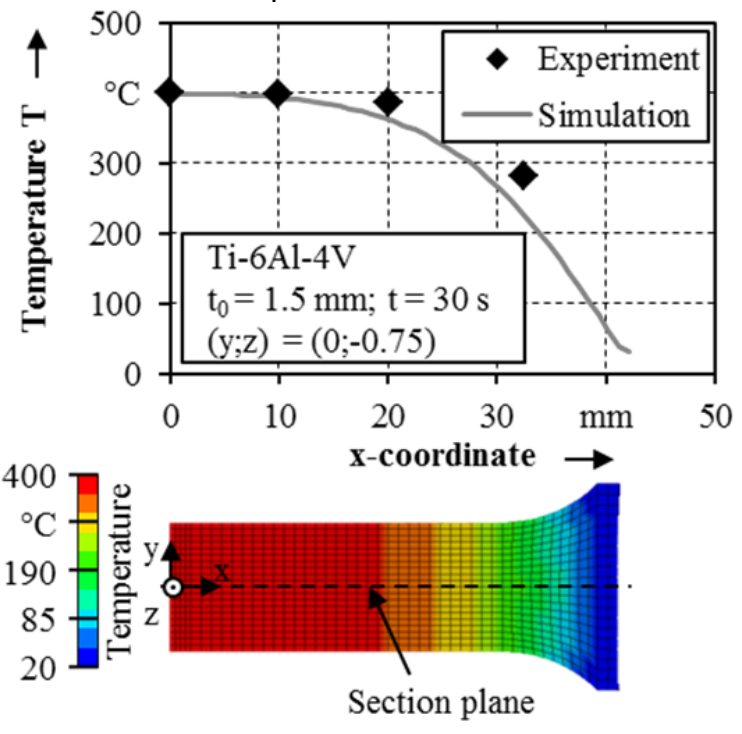

Fig. 6. Temperature distribution along the $\mathrm{x}$-axis

\subsubsection{Mechanical model}

Based on the electro-thermal simulation, the specimen is subdivided into four sections with different temperature fields. For each section different flow curves are assigned in the mechanical model thus emulating the thermal gradient of the real forming process. To show the influence of the thermal gradient the results are compared to an isothermal simulation with only one flow curve at $400{ }^{\circ} \mathrm{C}$. The mechanical model is validated by two criteria. On the one hand the results of the simulation are compared to the force-displacement curve from the experiment and on the other hand, the strain distribution along the longitudinal axis is taken into account. To show the influence of isothermal conditions the comparison is done for the specimen with temperature gradient subdivision and the isothermal sample. Fig. 7 shows the force-displacement curve for experiment and simulations including the maximum force. The elastic part of the curve, which is represented by a linear rise of the force is not fitted well by both simulations. One explanation could be that the elastic deformation of tool and machine of the experimental setup is neglected in the simulation. After the elastic part, both simulations show lower forces compared to the experiment. However, for the simulation with temperature dependent flow curves, the maximum force is reached for shorter displacement values, which fits the experimental results in a better way. For the simulation with isothermal conditions, the displacement at maximum force is much higher and therefore the deviations from experimental data as well.

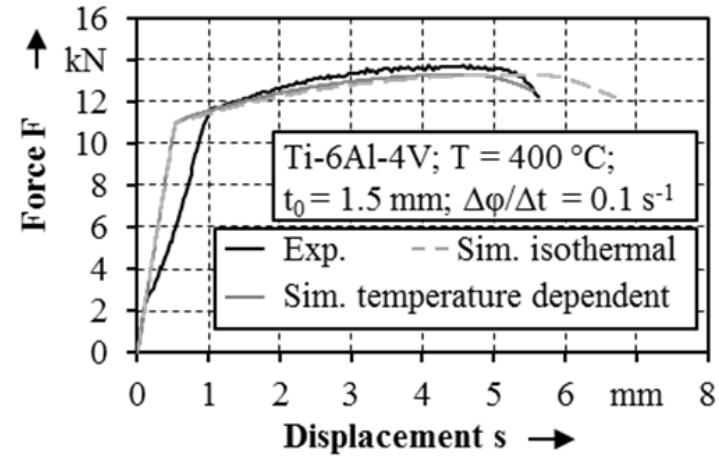

Fig. 7. Force-displacement for experiment and simulation

Based on these results, the force-displacement curve can be fitted better by using the gradient specimen, when the deviations in the elastic region are neglected. For a comprehensive investigation, the strain distribution along the longitudinal axis is evaluated at the point of maximum force, which is shown for the experiment and both simulations in Fig. 8 .

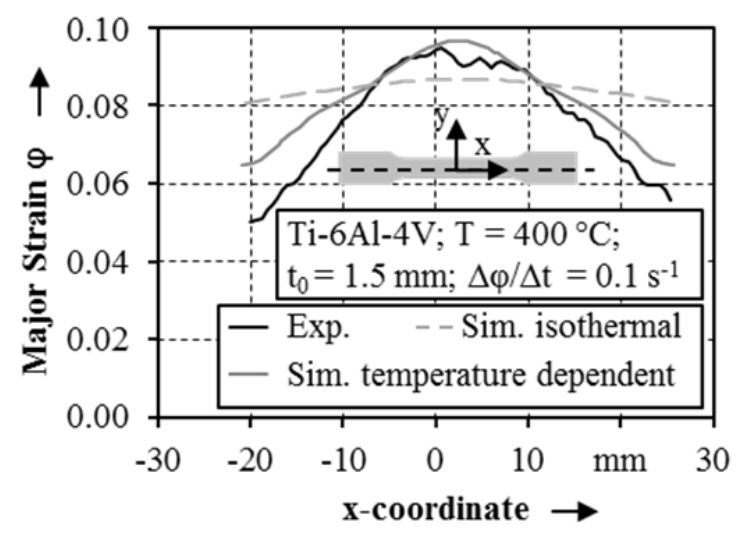

Fig. 8. Strain distribution along the $x$-axis

The deviation from the experimental data of maximum major strain 0,094 is $2.1 \%$ for the simulation with gradient properties 0,096 . Whereas, the deviation is $7.5 \%$ in case of isothermal conditions 0,087 . However, under isothermal conditions, the major strain is underestimated, which is crucial, because the real strains are higher. Besides the underestimation of the maximum strains, the simulation with temperature dependent flow curves calculates the strain distribution along the $\mathrm{x}$ coordinate of the experiment more precisely. Again the simulation with temperature gradient dependent flow curves shows a much better representation of the experimental data. This shows that the forming behaviour is strongly influenced by the temperature gradient. Therefore, the temperature gradient should be considered in simulations of hot forming processes. To 
handle non-uniform temperatures, which may occur during a hot forming process, the temperature deviation from the target temperature should be small.

\section{Conclusion}

The major findings of this work are that the temperature distribution and the strain behaviour can be simulated by simple electro-thermal and mechanical numerical models in LS-Dyna. With this methodology, the determination of mechanical properties in a resistance heating process can be supported. It can be clearly seen that the forming behaviour depends strongly on the temperature distribution. When using a homogeneous temperature in the forming process, the maximum major strain is underestimated, whereas the experimental results are fitted in good approximation by the temperature dependent mechanical model. This means more real forming operations at elevated temperature as warm bending or deep drawing of Ti-6Al-4V should consider the temperature distribution on the specimen during the forming process.

\section{Summary and Outlook}

In this work, the influence of a temperature distribution on the forming behaviour of Ti-6Al-4V for forming at elevated temperature is demonstrated with a tensile test at $400{ }^{\circ} \mathrm{C}$ by electro-thermal and mechanical numerical models using LS-Dyna and experimental results. As an experimental input, tensile tests at elevated temperature are conducted with a Gleeble 3500 with resistance heating. During the test, the current through the specimen is measured as an input parameter for the simulation. Afterwards, the mesh with the temperature distribution is subdivided into several sections with different flow curves and implemented into a mechanical model to investigate the temperature-dependent flow behaviour. By using the models, the temperature distribution after the heating process, the forcedisplacement-curve and the strain behaviour approximately represent the experimental data. To evaluate the influence of the temperature gradient, the results are compared to a sample with homogeneous material properties. With the temperature gradient specimen, the force-displacement-curve and the strain distribution is calculated more realistic. For the homogeneous material, the maximum major strain is underestimated. Hence, the forming behaviour is strongly influenced by the temperature gradient during the tensile test at elevated temperature.

Within further investigations, physical effects as convection and radiation should be implemented into the numerical models, which could reduce the deviations in temperature distribution. Furthermore, temperature dependent physical properties as thermal and electrical conductivity should be considered. Since the electrothermal and the mechanical model for the use of the temperature dependent material properties represent the experimental result, a coupled numerical model including heating and forming should be set up.
The authors acknowledge the support by German Research Foundation within CRC 814 - "Additive Manufacturing" subproject B5.

\section{References}

1. M. Peters, C. Leyens, Titanium and Titanium Alloys (2003)

2. J. Beal, R. Boyer, D. Sanders, Metalworking: Sheet Forming 656-669 (2006)

3. M. Merklein, H. Hagenah, M. Kaupper, A. Schaub, Key Engineering Materials 549 311-316 (2013)

4. S. Novotny, M. Celeghini, M. Geiger, Proc. of SheMet Int. Conf. 363-370 (2000)

5. K. Müller, Werkstoffkundliche Qualifizierung des Randschichthärtens mit Laserstrahlung (1999)

6. DIN EN ISO 6892-2, Metallic materials - Tensile testing part 2 (2017)

7. R. S. Lee, Y. K. Lin, T. W. Chien, Procedia Engineering 81 1682-1688 (2014)

8. M. Ganapathy, N. Li, J. Lin, M. Abspoel, H. Guido, D. Bhattacharjee, Matec Web of Conferences 21 (2015)

9. E. Kardoulaki, J. Lin, D. Balint, D. Farrugia, Journal of Strain Analysis 49 521-532 (2014)

10. K. Gerhardt, Untersuchung zur konduktiven Erwärmung für Warmzugversuche an Blechen (2015)

11. A. Schaub, J. Degner, A. Bhrigu, M. Schmidt, M. Merklein, Int. Conf. on Competitive Manufacturing, (2016)

12. M. Merklein, P. Dubjella, A. Schaub, L. Butzhammer, M. Schmidt 6th Int. Conf. on Additive Technologies 309-316 (2016)

13. A. Krawczynska, M. Lewandowska, R. Kuziak, K. Kurzydlowski, Phys. Status Solidi 5 1380-1383 (2010)

14. P. Eplattenier, G. Cook, C. Ashcraft, M. Burger, J. Imbert, M. Worswick Steel Research Int. 5 351-358 (2009)

15. P. Eplattenier, I. Caldichoury, Int. LS-Dyna Users Conf. 1-10 (2012)

16. LSTC, LS-Dyna Keywords Users's Manual R9.0 Material Models (2016)

17. M. Li, S. Zinkle, Comprehensive Nuclear Materials 4 667-690 (2012)

18. J. Valencia, P. Quested, ASM Handbook 15 468-481 (2008)

19. H. Swift, Journal of the Mechanics and Physics of Solids 1 1-18 (1952)

20. S. Nemat-Nasser, W. Guo, V. Nesterenko, S. Indrakanti Mechanics and Materials 33 425-439 (2001)

21. J. Hockett, O. Sherby, Journal of Phys. Solids 23 87-98 (1975) 